\title{
THE PRESENT STATE OF THE n-BODY PROBLEM
}

\author{
H. POLLARD
}

Purdue University, Ind., U.S.A.

The development of the $n$-body problem has occurred along four lines:

Determination of collision singularities. The main results here are due to von ZeipelSperling, and Pollard-Saari who have found necessary and sufficient conditions that singularities be due to collisions.

Behavior of the system as $t \rightarrow \infty$. The main results are due to Pollard and more especially Saari, and are concerned with the long behavior of a system unimpaired by singularities.

The topology of the solution surfaces. Smale has examined the differential equations by modern topological methods. The results which are satisfactory in the case of planar motion, still need development in the general case.

Numerical results. The results, due to a number of investigators, are far from definitive. 